\title{
Comparison of Early and Delayed Inpatient Dexamethasone Suppression Tests
}

\author{
Roger F. Haskett, Athanasios P. Zis, A. Ariav Albala, Naomi E. Lohr, and \\ Bernard J. Carroll
}

Received August 10. 1987; revised version received October 17, 1988; accepted December 17. 1988.

\begin{abstract}
Ninety-five inpatients completed a dexamethasone suppression test (DST) within 72 hours after admission and again after at least 1 week of medication-free hospital care. The frequency of cortisol nonsuppression in patients with endogenous depression (ED) was high and not significantly different at both tests. In patients with diagnoses other than ED, the higher rate of cortisol nonsuppression at the first DST was associated with a significant decrease in test specificity. Change in postdexamethasone cortisol levels at repeat testing was associated with a decrease in depressive symptomalology, but was not related to weight change during hospitalization.
\end{abstract}

Key Words. Depression, dexamethasone, cortisol, inpatients.

Several reviews of the literature have concluded that, in certain specific situations, the dexamethasone suppression test (DST) can contribute to the diagnostic evaluation of depression (Carroll, 1982; Arana et al., 1985; APA Task Force on Laboratory Tests in Psychiatry, 1987). Although the controversy associated with this topic has many sources (Carroll, 1985), some of the discrepancies in reported findings have been associated with the spread of DST studies into general clinical settings. Most early studies examined the performance of the DST in patients on research units; the patients usually were drug free and had been hospitalized for 1-2 weeks before the test was performed. These restrictions tend to emphasize the specific effects of depressive illness on the hypothalamic-pituitary-adrenal (HPA) axis and to diminish the nonspecific effects of other factors that activate this endocrine system. These factors include "psychological stress" (Mason, 1968; Blumenfield et al., 1970), admission to the hospital (Mason et al., 1965; Sachar, 1967; Swigar et al., 1979), and withdrawal from medications (Devanand et al., 1984; Dilsaver and Greden, 1985) or acute alcohol withdrawal (Swartz and Dunner, 1982;

Roger F. Haskett, M.B., B.S., is Associate Professor of Psychiatry, and Naomi E. Lohr, Ph.D., is Assistant Professor of Psychology, Department of Psychiatry, University of Michigan, Ann Arbor, MI 48109. Athanasios P. Zis, M.D., is Professor of Psychiatry, Department of Psychiatry, University of British Columbia, Vancouver, BC V6T 2A1, Canada. A. Ariav Albala, M.D., is Associate Clinical Professor of Psychiatry, Department of Psychiatry, University of California, San Diego, La Jolla, CA 92093. Bernard J. Carroll, M.B., Ph.D., is Professor and Chairman, Department of Psychiatry, Duke University Medical Center, Durham, NC 27710. (Reprint requests to Dr. R.F. Haskett, Dept. of Psychiatry, D9702, Box 0118, University of Michigan Medical Center, Ann Arbor, MI 48109-0118, USA.) 
Newsom and Murray, 1983; Ravi et al., 1984; DelPorto et al., 1985).

Although many psychiatrists in clinical practice have ordered the DST promptly after the patient's hospital admission, we cannot assume that the DST will provide optimal diagnostic information when used in this way. In an earlier study (Carroll et al., 1981), we reported no significant drop in sensitivity of the DST for melancholic inpatients as the drug-free period of observation was extended from 1-3 days to at least 10 days. No comparable data on nonmelancholic patients were discussed. Thus, although the sensitivity of the DST was unrelated to the length of time in the hospital, it does not necessarily follow that the same is true for the specificity of the test. A retrospective examination of case records (Coccaro et al., 1984) revealed that depressed patients who completed a DST on day 2 of hospitalization had a higher frequency of cortisol nonsuppression (71\%) than depressed patients who were tested on days 3-6 (33\%). This difference did not appear to result from an association between the severity of depression and the day of DST completion. A similar review of 42 depressed patients (Roy-Byrne et al., 1984) found a nonsignificant trend toward more frequent cortisol nonsuppression when patients were tested on days 1 or 2 of hospitalization compared with days 4,5 , or 6 . By contrast, the results obtained in depressed patients were highly replicable in another study when the DST was completed on days 8 and 15 of hospitalization (Charles et al., 1982).

In this study, we completed a DST first within 72 hours of hospitalization and again in the same patients after a medication-free evaluation period. This design permitted the assessment of any "admission effect" on the sensitivity, specificity, and predictive validity of the DST, as well as the test-retest reliability during the first 3 weeks of inpatient care.

\section{Methods}

Patients. This study was conducted on a 22-bed general psychiatric unit and included 95 consecutively admitted patients with the prominent symptom of depressed mood. On this unit, it was standard practice to discontinue all psychotropic medications gradually during the first week of hospitalization and complete a drug-free diagnostic evaluation during the subsequent week. Patients were excluded from the study if they were less than $80 \%$ of ideal body weight, suffered from physical illness, were taking medications known to affect the DST (Carroll et al., 1981), or were unable to complete the drug-free evaluation. Diagnostic evaluation included a structured interview using the Schedule for Affective Disorders and Schizophrenia (SADS) (Endicott and Spitzer, 1978) and used all available history and clinical observations. The diagnosis, according to Research Diagnostic Criteria (RDC) (Spitzer et al., 1978), was determined at the completion of the evaluation period by a consensus of the treating psychiatrist and the SADS interviewer.

We studied 58 women and 37 men whose ages were $40.6 \pm 15.3$ years and $30.0 \pm 10.0$ years, respectively (mean $\pm \mathrm{SD}$ ). Sixty-two patients were diagnosed as suffering from a depressive disorder hy R DC. Thirty-seven of these met criteria for major depressive disorder (MDD), definite endogenous subtype (ED). The remaining 25 met criteria for MDD, not or only probable endogenous subtype; episodic minor depressive disorder; or chronic and intermittent depressive disorder (NED). The ratio of females to males was 2:1 in both ED and NED groups, and the mean ages were 43.6 years (range $21-73$ ) and 32.7 years (19-60), respectively. The remaining patients received diagnoses of manic disorder (MAN) $(n=11)$; schizoaffective disorder, depressed type (SA-D) $(n=10)$; schizoaffective disorder, manic type (SA-M) $(n=7)$; or schizophrenia $(\mathrm{SCH})(n=5)$. 
Procedure. All patients completed a DST on two occasions before beginning treatment. Patients received dexamethasone at 11:30 p.m. on day 1 or 2 of inpatient care (DST-I), and plasma cortisol was measured at 4 p.m. and 11 p.m. on the following day. The test was repeated after 7-21 days of hospitalization (DST-II). DST-I could therefore have occurred in the absence of recent medication ingestion, during the discontinuation of known medications, or following the abrupt discontinuation of alcohol, marijuana, or undisclosed medications. DST-II always occurred at least 7 days after cessation of medication and after at least 1 week in the hospital. The DST followed the procedure described by Carroll et al. (1981), using $1 \mathrm{mg}$ of dexamethasone, and the result was considered nonsuppressive/abnormal if either the 4 p.m. or 11 p.m. postdexamethasone plasma cortisol value exceeded $5 \mu \mathrm{g} / \mathrm{dl}$. Plasma cortisol was determined using a competitive protein-binding method (Wilens et al., 1984). In our laboratory, the typical coefficients of variation are $12.5 \%$ (interassay) and $6.5 \%$ (intra-assay) with low cortisol samples (mean cortisol concentration, $2.5 \mu \mathrm{g} / \mathrm{dl}$ ), and $9.0 \%$ (interassay) and $5.5 \%$ (intra-assay) with high cortisol samples (mean cortisol concentration, $10.0 \mu \mathrm{g} / \mathrm{dl}$ ).

Clinical ratings of severity were obtained at the time of each DST using the Hamilton Rating Scale for Depression (HRSD) (Hamilton, 1960). The results of the DST were not released to the investigators until the diagnostic evaluation was complete.

Data Analysis. Two sets of analyses were performed on the dependent measures derived from the DST results. First, we calculated the frequency of "DST nonsuppression" in each diagnostic group and examined changes in test performance between DST-I and DST-II. The significance of a change in frequency between DST-I and DST-II was assessed using the McNemar $\chi^{2}$ test for dependent samples (McNemar, 1947). The 95\% confidence limits (CL95) for estimates of conditional probabilities (sensitivity, specificity, and predictive validity) were determined as described previously (Kalter et al., 1983). A Kappa statistic was calculated for each patient group as a measure of agreement in test result between DST-1 and DST-II (Cohen, 1960).

In a separate analysis, we examined the actual change in maximum postdexamethasone cortisol levels at DST-I and DST-II. The cortisol values were log-transformed to improve the normality of distribution before the use of parametric statistical tests (skewness at DST-I and DST-II, respectively, was 0.97 and 1.32 for raw cortisol values and -0.73 and 0.24 after $\log$ transformation). To explore the relationship between diagnosis and change in postdexamethasone cortisol values, age, weight, and HRSD score, we used a two-way analysis of variance (ANOVA) with diagnosis as a grouping factor and time as the repeated measures dimension or a one-way ANOVA as appropriate. The associations between change in postdexamethasone cortisol values and patient's age, weight, and HRSD score were studied by analyses of covariance (ANCOVAs).

\section{Results}

Table 1 shows that the frequency of abnormal DST results in all studied patients significantly decreased from $55 \%$ at DST-I to $36 \%$ at DST-II $(p<0.01)$. Although the sensitivity (i.e., frequency of an abnormal DST in patients with ED) also appeared to decrease from $70 \%$ at DST-I to $59 \%$ at DST-11, the presence of a clear overlap in the CL-95 for each estimate (55\%-85\% and 43\%-75\%, respectively) indicates that this change is inconclusive. By comparison, the overall specificity (i.e., frequency of a normal DST in patients with diagnoses other than ED) increased from $55 \%$ at DST-I to $79 \%$ at DST-II, and there was no overlap in the CL-95 for each estimate $(45 \%-65 \%$ and $71 \%-87 \%$, respectively).

The agreement in test results between DST-I and DST-II also tended to be higher in patients with ED than in other patients. The value of Kappa for the ED patients was 0.53 , and in the remaining patients it was 0.19 . The CL-95 for the difference 
Table 1. Frequency of dexamethasone suppression test (DST) nonsuppression and test-retest reliability within diagnostic groups

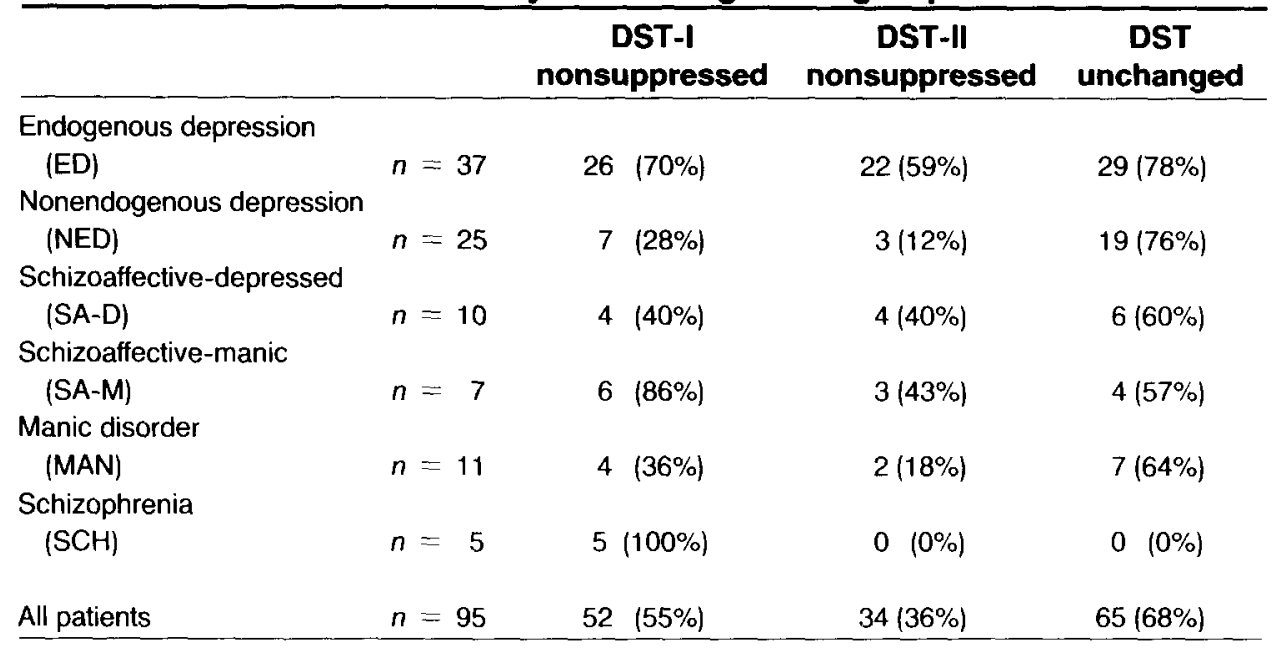

in these Kappa values is $0.34 \pm 0.38$, which just overlaps zero. $A$ one-sided $t$ test gives a $p$ value of 0.04 . Further examination of ED patients who also met psychotic subtype revealed that $92 \%$ of DST results were unchanged $(12 / 13)$ and $77 \%(10 / 13)$ were abnormal at both times. Eight patients also met criteria for alcoholism during the index episode, and three had an abnormal result at DST-I (their concurrent diagnoses were ED, SA-D, and $\mathrm{SCH}$ ). None developed a clinically evident withdrawal syndrome, and all suppressed cortisol normally at DST-II.

Table 2 summarizes the mean postdexamethasone cortisol levels for each diagnostic group. Similar to the frequency of nonsuppression, the actual cortisol levels decreased in all diagnostic groups between DST-I and DST-II. In the ED patients, however, the mean cortisol level at DST-II remained higher than the criterion for nonsuppression, while the comparable value in patients with NED, $\mathrm{MAN}$, or $\mathrm{SCH}$ fell bclow the criterion. Because of small numbers of patients in many

Table 2. Maximum postdexamethasone cortisol levels at DST-I and DST- $I I(\mu / d I$, mean \pm SD)

\begin{tabular}{crrr}
\hline Diagnosis & $\boldsymbol{n}$ & DST-I & DST-II \\
\hline ED & 37 & $11.2+8.2$ & $8.5+6.7$ \\
NED & 25 & $3.9 \pm 4.8$ & $2.8 \pm 3.0$ \\
SA-D & 10 & $5.9 \pm 5.9$ & $4.1 \pm 3.6$ \\
SA-M & 7 & $12.2 \pm 6.8$ & $6.1+5.5$ \\
MAN & 11 & $6.4 \pm 5.8$ & $2.8 \pm 3.3$ \\
SCH & 5 & $10.9 \pm 5.0$ & $2.3 \pm 1.4$ \\
OTHDEPR & 35 & $45 \pm 5.1$ & $31+32$ \\
OTHER & 58 & $6.3 \pm 6.0$ & $3.3 \pm 3.5$ \\
\hline
\end{tabular}

Note. DST $=$ dexamethasone suppression test. $E D=$ endogenous depression. $\mathrm{NED}=$ nonendogenous depression. SA-D - schizoaffective disorder, depressed. SA-M = schizoaffective disorder, manic. SCH = schizophrenia. OTHDEPR = patients with other depressive syndromes (i.e., NED or SA-D). OTHER = all other patients (i.e., NED, SA-D, SA-M, MAN, or SCH). 
of the nondepressed diagnostic groups, statistical analyses were confined to comparisons between patients with ED and all other patients (OTHER = NED, SA-D, SA-M, MAN, and SCH) or between patients with ED and patients with other depressive syndromes (OTHDEPR $=$ NED or SA-D). All of the OTHER group presented with depressed mood, whereas the OTHDEPR group actually had a depressive syndrome. Comparison of postdexamethasone cortisol levels between these patient groups revealed significant main effects for diagnosis (ED:OTHER, $p<0.0001$; ED:OTHDEPR, $p<0.0001$ ) and time (ED:OTHER, $p<0.0001$; ED:OTHDEPR, $p=0.02$ ), but the time $\times$ diagnosis interaction was not significant (ED:OTHER, $p=0.5$; ED:OTHDEPR, $p=0.6$ ). From these results, it appears that the postdexamethasone cortisol levels were significantly higher in the ED patients, that cortisol levels decreased significantly between tests across diagnoses, and that the magnitude of the decrease was not significantly different between the ED patients and either comparison group.

Although patients in the ED group were significantly older than patients in the OTHER and OTHDEPR groups ( $p=0.0001$ and $p=0.0001$, respectively), age was not found to be a significant covariate on the cortisol level between diagnoses (ED:OTHER, $p=0.5$; ED:OTHDEPR, $p=0.3$ ).

Comparison of HRSD scores between diagnostic groups, and between DST-I and DST-II, revealed significant main effects for group (ED:OTHER, $p<0.0001$; ED:OTHDEPR, $p<0.0001$ ) and time (ED:OTHER, $p=0.0001$; ED:OTHDEPR, $p=0.001$ ), but the time $\times$ diagnosis interaction was not significant (ED:OTHER, $p=0.5$; ED:OTHDEPR, $p=0.7$, respectively). There was a trend for the HRSD score to contribute to the between group differences in cortisol level (ED:OTHER, $p=0.09$; ED:OTHDEPR, $p=0.07$ ). After the HRSD effect was controlled for, however, the main effect for diagnosis continued to have a significant effect on the variance in cortisol level (ED:OTHER, $p=0.006$; ED:OTHDEPR, $p=0.002$ ). When all patients in the study were considered, the HRSD score was a significant covariate on the differences in cortisol level between tests (ED:OTHER, $p=0.03$ ), and after the HRSD score was controlled for, the main effect for time was no longer significant (ED:OTHER, $p=0.09$ ). If only the patients with a depressive syndrome were considered, the HRSD score did not significantly contribute to the withinsubject variation in cortisol level between the two tests (ED:OTHDEPR, $p=0.03$ ). These results suggest that patients with ED had significantly more severe depressive symptomatology and that, although there was a significant decrease in severity between tests, the magnitude of the decrease was not significantly different between the ED patients and either comparison group. Although the HRSD score may contribute to the cortisol level differences between diagnostic groups, other factors are clearly important. The effect of the decrease in HRSD score between tests on the cortisol levels appears to be significant when all patients are considered in contrast to the nonsignificant effect when only patients with depressive syndromes are examined.

A similar analysis of patient weights revealed no significant main effects for group (ED:OTHER, $p=0.07$ and ED:OTHDEPR, $p=0.8$ ) or time (ED:OTHER, $p=0.4$ and ED:OTHDEPR, $p=0.1$ ). (See Table 3.) 
Table 3. Age, weight, and HRSD score at DST-I and DST-II (mean \pm SD)

\begin{tabular}{cccccr}
\hline Diagnosis & Age (years) & \multicolumn{2}{c}{ Weight (lbs) } & \multicolumn{2}{c}{ HRSD } \\
\hline ED & $43.6 \pm 15.4$ & $153 \pm 35$ & $153 \pm 34$ & $26 \pm 7$ & $23 \pm 7$ \\
NED & $32.4 \pm 11.1$ & $145 \pm 27$ & $147 \pm 28$ & $16 \pm 7$ & $12 \pm 7$ \\
SA-D & $26.9 \pm 5.0$ & $163 \pm 35$ & $164 \pm 35$ & $18 \pm 12$ & $16 \pm 9$ \\
SA-M & $26.7 \pm 6.8$ & $158 \pm 49$ & $\mathbf{1 6 0} \pm 47$ & $14 \pm 5$ & $9 \pm 5$ \\
MAN & $42.7 \pm 14.5$ & $153 \pm 36$ & $151 \pm 36$ & $9 \pm 5$ & $7 \pm 5$ \\
SCH & $22.2 \pm 3.0$ & $135 \pm 22$ & $137 \pm 23$ & $17 \pm 5$ & $9 \pm 5$ \\
OTHDEPR & $30.9 \pm 10.0$ & $150 \pm 30$ & $152 \pm 30$ & $16 \pm 8$ & $13 \pm 8$ \\
OTHER & $31.9 \pm 11.7$ & $151 \pm 33$ & $151 \pm 33$ & $15 \pm 8$ & $11 \pm 7$ \\
\hline
\end{tabular}

Note. HRSD = Hamilton Rating Scale for Depression. DST = dexamethasone suppression test. ED = endogenous depression. NED = nonendogenous depression. $S A-D=$ schizoaffective disorder, depressed. $S A-M=$ schizoaffective disorder, manic. $\mathrm{SCH}=$ schizophrenia. OTHDEPR = patients with other depressive syndromes (i.e., NED or SA-D). OTHER = all other patients (i.e., NED, SA-D, SA-M, MAN, or SCH).

On the basis of the medication history obtained at the time of admission, patients were categorized as medication free or withdrawn from one of the common classes of psychotropic medications, ethanol, or cannabis (Table 4). Many patients were withdrawn from multiple medications, and therefore the sum of the percentages is $>100 \%$. When patients with ED were compared to all other patients (OTHER), it was apparent that the more frequent change from nonsuppression to suppression in the OTHER group could not be clearly related to withdrawal from any specific group of medications.

Table 4. Preadmission medication history

\begin{tabular}{lcrrrrrc}
\hline & ED & OTHER & \multicolumn{1}{c}{ NED } & SA-D & SA-M & MAN & SCH \\
\hline Medication free & $10(27 \%)$ & $23(40 \%)$ & $11(44 \%)$ & $4(40 \%)$ & $3(43 \%)$ & $4(36 \%)$ & $1(20 \%)$ \\
Antidepressants & $17(46 \%)$ & $9(16 \%)$ & $7(28 \%)$ & $0(0 \%)$ & $0(0 \%)$ & $2(18 \%)$ & $0(0 \%)$ \\
Neuroleptics & $11(30 \%)$ & $20(34 \%)$ & $3(12 \%)$ & $4(40 \%)$ & $4(57 \%)$ & $5(45 \%)$ & $4(80 \%)$ \\
Antiparkinsonian & $2(5 \%)$ & $6(10 \%)$ & $1(4 \%)$ & $2(20 \%)$ & $1(14 \%)$ & $0(0 \%)$ & $2(40 \%)$ \\
Benzodiazepines & $7(19 \%)$ & $4(7 \%)$ & $3(12 \%)$ & $0(0 \%)$ & $0(0 \%)$ & $1(9 \%)$ & $0(0 \%)$ \\
Lithium & $1(3 \%)$ & $5(9 \%)$ & $0(0 \%)$ & $1(10 \%)$ & $0(0 \%)$ & $3(27 \%)$ & $1(20 \%)$ \\
Ethanol/cannabis & $5(14 \%)$ & $7(12 \%)$ & $6(24 \%)$ & $1(10 \%)$ & $0(0 \%)$ & $0(0 \%)$ & $0(0 \%)$ \\
Miscellaneous & $0(0 \%)$ & $4(7 \%)$ & $3(12 \%)$ & $0(0 \%)$ & $1(14 \%)$ & $0(0 \%)$ & $0(0 \%)$ \\
Total & $(143 \%)$ & $(134 \%)$ & $(136 \%)$ & $(120 \%)$ & $(129 \%)$ & $(136 \%)$ & $(160 \%)$ \\
$\quad$ S to NS & $2(5 \%)$ & $4(7 \%)$ & $1(4 \%)$ & $2(20 \%)$ & $0(0 \%)$ & $1(9 \%)$ & $0(0 \%)$ \\
NS to S & $6(16 \%)$ & $18(31 \%)$ & $5(20 \%)$ & $2(20 \%)$ & $3(43 \%)$ & $3(27 \%)$ & $5(100 \%)$ \\
\hline
\end{tabular}

Note. $\mathrm{S}=$ dexamethasone suppression test (DST) suppression. NS = DST nonsuppression. ED = endogenous depression. OTHER $=$ all other patients. NED $=$ nonendogenous depression. SA-D $=$ schizoaffective disorder, depressed. SA-M = schizoaffective disorder, manic. SCH = schizophrenia.

\section{Discussion}

Our principal finding was that completion of the DST within 72 hours of admission produced a higher frequency of cortisol nonsuppression than occurred when the DST was delayed until week 2 of hospitalization after the patients had been medication free for 1 week. Although the frequency of abnormal results at DST-I was increased in most diagnoses, the difference between DST-I and DST-II was 
inconclusive in the 37 patients with ED. This is in agreement with our previous report (Carroll et al., 1981) of no significant change in DST sensitivity for ED after hospitalization and withdrawal from medication. In patients with diagnoses other than ED, however, the increase in frequency of abnormal results of DST-I was associated with a significant decrease in test specificity.

The change in frequency of cortisol nonsuppression between DST-I and DST-II also altered the predictive value of an abnormal DST for ED ( $P V+)$ (percentage of patients with an abnormal DST result having a diagnosis of ED). Administration of the DST to all newly admitted patients resulted in a PV+ of 50\% (CL-95 $=40 \% \%^{-}$ $60 \%$ ). When the test was administered to the same patients after at least 1 week free of medication and after at least 1 week in the hospital, the PV+ increased to 65\% $(\mathrm{CL}-95=55 \%-75 \%)$. Since PV+ is also dependent upon the prevalence of ED in the tested group (Vecchio, 1966; Baldessarini et al., 1983), further improvement can be achieved by increasing the expected prevalence of ED in the sample. If the second test had been administered to only those patients with depressive syndromes (ED, NED, and SA-D), the PV+ would have been $76 \%$ (CL-95 $=66 \%-86 \%$ ); after 1 week in hospital and medication free, nearly 8 out of every 10 depressed patients with an abnormal result at DST-II met diagnostic criteria for ED.

The results also reinforce the earlier warning about interpretation of DST results in newly admitted psychotic patients (Carroll et al., 1976). This caution is supported by the report of Herz et al. (1985), who found cortisol nonsuppression in $73 \%$ of patients with schizophrenia or schizoaffective disorder when the DST was completed on days 1 and 2 of hospitalization. In most of their patients, the DST result normalized after 1 week's treatment with neuroleptic medication. Our own results suggest that normalization of the DST in psychotic but nondepressed patients (SA$\mathrm{M}$ and $\mathrm{SCH}$ ) will frequently occur after $\mathbf{I}$ week of inpatient care without definitive drug treatment. This contrasts with the results from psychotic patients who also manifested a depressed syndrome (SA-D, ED-psychotic subtype); these patients had a similar frequency of cortisol nonsuppression at both times of testing.

In this sample, patients with MAN had a low frequency of cortisol nonsuppression, which was much lower than the frequency in patients meeting criteria for SA-M. Although other reports have noted a high frequency of cortisol nonsuppression in mania (Graham et al., 1982; Arana et al., 1983), data from studies that have used alternative diagnostic criteria (such as $D S M$ - $I I I$ or ICD-9) to categorize affective syndromes cannot be compared directly to studies that used the RDC (Davidson et al., 1984). Despite having the same "diagnoses," groups of manic patients diagnosed at different centers by varying diagnostic criteria will not be strictly comparable.

The factors responsible for the frequent change from cortisol nonsuppression at DST-I to suppression at DST-II cannot be comprehensively addressed in this study. Although physiological factors, such as inadequate hydration, poor food intake with associatcd kctosis, and disrupted circadian rest-activity cycles, may be suspected (Carroll, 1984), drug withdrawal is another possibility. Rapid withdrawal of medication with significant anticholinergic activity is sometimes associated with activation of the HPA axis (Dilsaver and Greden, 1985). Many patients were being 
withdrawn from various psychotropic medications during DST-I, whereas all had been medication free for at least I week before DST-II. Despite the significant decrease in frequency of cortisol nonsuppression in patients with diagnoses other than ED, there does not appear to be any particular class of medications that was most commonly discontinued after admission in these patients. Psychotropic medications vary greatly in their pharmacological properties, and for an appropriate examination of this interaction, sufficient numbers of patients with the same diagnoses and taking single medications should have them discontinued.

Although the frequency of cortisol nonsuppression in the ED patients did not decrease significantly between tests, there was a significant decrease in the absolute postdexamethasone cortisol level. The magnitude of this decrease in the ED patients, however, did not significantly differ from that seen in other depressed patients or in all other patients. This suggests that the factors associated with a decreased response of the HPA axis to dexamethasone at DST-I compared to DST-II (i.e., more nonsuppression), did not appear to affect patients with ED differentially as compared to patients with other diagnoses. The lack of plasma dexamethasone levels at DST-I and DST-II prevented us from examining the contribution of changes in dexamethasone pharmacokinetics to this altered HPA axis response. It is notable that a significant fall in the postdexamethasone cortisol levels in the ED group did not produce a significant change in the frequency of nonsuppression in this diagnostic group. Inspection of the mean values in Table 2 indicates that an equivalent decrease in absolute postdexamethasone cortisol levels in the ED group did not have the same impact on the frequency on nonsuppression because, in these patients, most values still remained above the $5 \mu \mathrm{g} / \mathrm{dl}$ criterion.

Several studies have found a positive correlation between age and postdexamethasone cortisol levels in depressed patients (Asnis et al., 1981; Lewis et al., 1984; Stokes et al., 1984). Although our patients with ED were significantly older than the patients with other diagnoses, ANCOVA revealed that the betweendiagnosis difference in the postdexamethasone cortisol levels remained significant after controlling for the age difference.

Similarly, there have been reports indicating that severity of depressive symptomatology is positively associated with cortisol nonsuppression (Miller and Nelson, 1987; Meador-Woodruff et al., 1988). In this study, it was expected that more severe depressive features would be present in patients with ED, but even after the difference in severity of depression was controlled for, postdexamethasone cortisol levels in ED patients remained significantly higher than the levels in patients with other diagnoses. Our results also documented a modest but generalized decrease in symptomatology during the medication-free evaluation. The beneficial effect of the inpatient milieu, although sometimes not sustained, has been commonly observed in patients with a wide range of presentations. Once this decrease in HRSD score was controlled for, the fall in postdexamethasone cortisol levels between DST$I$ and DST-Il was no longer significant, suggesting that the improvement in symptomatology and the decrease in cortisol levels were closely associated. Contrary to earlier suggestions that weight loss might be responsible for abnormal DST results (Berger et al., 1983; Edelstein et al., 1983), we found no significant relationship 
between change in weight and change in postdexamethasone cortisol level in the hospital.

In conclusion, our results indicate that abnormal DST results are common but transient among nondepressed psychotic patients during the first 3 days after hospital admission. Nonsuppression rates are also elevated soon after admission in patients with mania and NED, though to a lesser extent than in psychotic patients. In patients with ED, a high rate of nonsuppression persisted well beyond the first days of hospitalization. The practical lesson from these results is that the diagnostic performance of the DST will be reduced if it is performed during the first few days after patients enter the hospital.

Acknowledgments. The authors thank Patricia Nelson, R.N., James Ritchie, M.S., Kathleen F. Johnson, B.A., and Pamela Flegel, B.S., for their invaluable assistance with this study. David Amato, Ph.D., provided advice on the statistical analysis. The research reported was supported in part by NIMH grant MH-28294.

\section{References}

APA Task Force on Laboratory Tests in Psychiatry. The dexamethasone suppression test: An overview of its current status in psychiatry. American Journal of Psychiatry, 144:1253-1262, 1987.

Arana, G.W.; Baldessarini, R.J.; and Ornsteen, M. The dexamethasone suppression test for diagnosis and prognosis in psychiatry. Archives of General Psychiatry. 42:1193-1204, 1985.

Arana, G.W.; Barreira, P.J.; Cohen, B.M.; Lipinski, J.F.; and Fogelson, D. The dexamethasone suppression test in psychotic disorders. American Journal of Psychiatry, 140:1521-1523, 1983.

Asnis, G.M.; Sachar, E.J.; Halbreich, U.; Nathan, R.S.; Novacenko, H.; and Ostrow, L.C. Cortisol secretion in relation to age in major depression. Psychosomatic Medicine, 43:235$242,1981$.

Baldessarini, R.J.; Finkelstein, S.; and Arana, G.W. The predictive power of diagnostic tests and the effect of prevalence of illness. Archives of General Psychiatry, 40:569-573, 1983.

Berger, M.; Pirke, K.M.: Doerr, P.; Krieg, C.; and von Zerssen, D. Influence of weight loss on the dexamethasone suppression test. Archives of General Psychiatry, 40:585-586, 1983.

Blumenfield, M.; Rose, L.I.; Richmond, L.H., and Beering, S.C. Dexamethasone suppression in basic trainees under stress. Archives of General Psychiatry, 23:299-304, 1970.

Carroll, B.J. The dexamethasone suppression test for melancholia. British Journal of Psychiatry, 140:292-304, 1982.

Carroll, B.J. Dexamethasone suppression test. In: Hall, R.C.W., and Beresford, T.P., eds. Handbook of Psychiatry: Diagnostic Procedures. Vol. I. Jamaica, NY: Spectrum, 1984. pp. 3-28.

Carroll, B.J. Dexamethasone suppression test: A review of contemporary confusion. Journal of Clinical Psychiatry, 46:13-24, 1985.

Carroll, B.J.; Curtis, G.C.; and Mendels, J. Neuroendocrine regulation in depression: II. Discrimination of depressed from nondepressed patients. Archives of General Psychiatry, 33:1051-1058, 1976.

Carroll, B.J.; Feinberg, M.; Greden, J.F.; Tarika, J.; Albala, A.A.; Haskett, R.F.; James, N.Mcl.; Kronfol, Z.; Lohr, N.; Steiner, M.; de Vigne, J.P.; and Young, E. A specific laboratory test for the diagnosis of melancholia. Archives of General Psychiatry, 38:15-22, 1981.

Charles, G.; Wilmotte, J.; Quenon, M.; and Mendlewicz, J. Reproducibility of the dexamethasone suppression test in depression. Biological Psychiatry. 17:845-848, 1982.

Coccaro, E.F.; Prudic, J.; Rothpearl, A.; and Nurnberg, H.G. Effect of hospital admission on DST results. American Journal of Psychiatry, 141:982-985, 1984. 
Cohen, J. A coefficient of agreement for nominal scales. Educational Psychological Measures, 20:37-46, 1960.

Davidson, J.; Lipper, S.; Zung, W.W.K., Stickland, R.; Krishnan, R.; and Mahorney, S. Validation of four definitions of melancholia by the dexamethasone suppression test. American Journal of Psychiatry, 141:1220-1223, 1984.

DelPorto, J.A.; Montiero, M.G.; Larajeira, R.R.; Jorge, M.R.; and Masur, J. Reversal of abnormal dexamethasone suppression test in alcoholics abstinent for four weeks. Biological Psychiatry, 20:1156-1160, 1985.

Devanand, D.P.; Pandurangi, A.K.; and Dewan, M.J. False-positive dexamethasone suppression test results related to antipsychotic drug withdrawal: Case report. Journal of Clinical Psychiatry, 45:275-276, 1984.

Dilsaver, S.C., and Greden, J.F. Effects of antidepressant withdrawal on the dexamethasone suppression test. Psychiatry Research, 14:111-122, 1985.

Edelstein, C.K.; Roy-Byrne, P.; Fawzy, F.I.; and Dornfeld, L. Effects of weight loss on the dexamethasone suppression test. American Journal of Psychiatry, 140:338-341, 1983.

Endicott, J., and Spitzer, R.L. A diagnostic interview: The Schedule for Affective Disorders and Schizophrenia. Archives of General Psychiatry, 35:837-844, 1978.

Graham, P.M.; Booth, J.; Gianfranco, B.; Galhenase, S.; Myers, C.M.; Teoh, C.L.; and Cox, L.S. The dexamethasone suppression test in mania. Journal of Affective Disorders, 4:201-211, 1982.

Hamilton, M. A rating scale for depression. Journal of Neurology, Neurosurgery and Psychiatry, 23:56-62, 1960.

Herz, M.I.; Fava, G.A.; Molnar, G.; and Edwards, L. The dexamethasone suppression test in newly hospitalized schizophrenic patients. American Journal of Psychiatry, 142:127-129, 1985.

Kalter, N.; Feinberg, M.; and Carroll, B.J. Inferential statistical methods for strengthening the interpretation of laboratory test results. Psychiatry Research, 10:207-213, 1983.

Lewis, D.A.; Pfohl, B.; Schlechte, J.; and Coryell, W. Influence of age on the cortisol response to dexamethasone. Psychiatry Research. 13:213-220, 1984.

Mason, J.W. A review of psychoendocrine research on the pituitary adrenal cortical system. Psychosomatic Medicine, 30:576-607, 1968.

Mason, J.W.; Sachar, E.J.; and Fishman, J.R. Corticosteroid responses to hospital admission. Archives of General Psychiatry, 13:1-8, 1965.

McNemar, Q. Note on the sampling error of the difference between proportions or percentages. Psychometrika, 12:153-157, 1947.

Meador-Woodruff, J.H.; Greden, J.F.; Grunhaus, L.; and Haskett, R.F. Dexamethasone suppression test status and severity of depression. Biological Psychiatry, 24:693-696, 1988.

Miller, K.B., and Nelson, J.C. Does the dexamethasone suppression test relate to subtypes, factors, symptoms, or severity? Archives of General Psychiatry, 44:769-774, 1987.

Newsom, G., and Murray, N. Reversal of dexamethasone suppression test nonsuppression in alcohol abusers. American Journal of Psychiatry, 140:353-354, 1983. 
Ravi, S.D.; Dorus, W.; Park, Y.N.; Cullins, M.C.; Reid, R.W.; and Borge, G.F. The dexamethasone suppression test and depressive symptoms in early and late withdrawal from alcohol. American Journal of Psychiatry, 141:1445-1448, 1984.

Roy-Byrne, P.; Gwirtsman, H.; Sternbach, H.; and Gerner, R.H. Effects of acute hospitalization on the dexamethasone suppression and TRH stimulation tests. Biological Psychiatry, 19:607-612, 1984.

Sachar, E.J. Corticosteroids in depressive illness: A re-evaluation. Archives of General Psychiatry, 17:544-553, 1967.

Spitzer, R.L.; Endicott, J.; and Robins, E. Research Diagnostic Criteria $(R D C)$ for a Selected Group of Functional Disorders. 3rd ed. New York: New York State Psychiatric Institute, 1978.

Stokes, P.E.; Stoll, P.M.; Koslow, S.H.; Maas, J.W.; Davis, J.M.; Swann, A.C.; and Robins, E. Pretreatment DST and hypothalamic-pituitary-adrenocortical function in depressed patients and comparison groups. Archives of General Psychiatry, 41:257-267, 1984.

Swartz, C.M., and Dunner, F.J. Dexamethasone suppression testing of alcoholics. Archives of General Psychiatry, 39:1309-1312, 1982.

Swigar, M.E.; Kalakowska, T.; and Quinlan, D.M. Plasma cortisol levels in depression and other psychiatric disorders: A study of newly admitted psychiatric patients. Psychological Medicine, 9:449-455, 1979.

Vecchio, T.J. Predictive value of a single diagnostic test in unselected populations. New England Journal of Medicine, 274:1171-1173, 1966.

Wilens, T.E.; Ritchie, J.C.; and Carroll, B.J. Comparison of plasma cortisol and corticosterone in the DST for melancholia. Psychoneuroendocrinology, 9:45-55, 1984. 